دراسة هيدرومورفولوجية بحيرة سد الموصل التنظيمية شمال مدينة الموصل، العراق

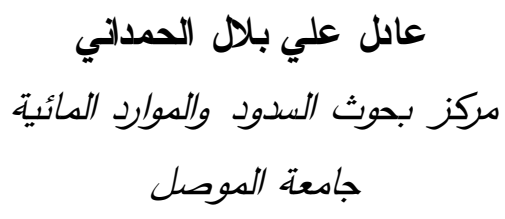

(تاريخ الاستلام 2014/3/5 تاريخ القبول 2014/4/9)

\author{
اسعد احمد مقداد آل حسين \\ قسم الجغرافية /كلية التربية \\ جامعة الحمدانية
}

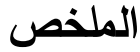

يتضمن البحث دراسة هيدرومورفولوجية البحيرة التنظيمية التي تقع على بعد (50km) شمالي مدينة الموصل. تمت عملية المسح في شهر تشرين الثاني من عام (2012) بأستخدام جهاز مسبار قياس الاعماق (EchoSounder)

وتم تسجيل البيانات وتمثيلها بأستخدام تقنية نظم المعلومات الجغرافية (Arc GIs v10.4) وبرنامج (Excel). تبين من خلال المسح ان اقل عمق في البحيرة وجد في الضفة اليسرى للمقطع العرضي (5) وبلغ واعمق نقطة وجلت في الضفة اليسرى للمقطع العرضي (9.56m) وبلغت (18.22m) على خط الثالوك. وجد ان انحدار البحيرة هو (1m/Km)، والثكل العام لأغلب المقاطع العرضية مشابه إلى الحرف (U).

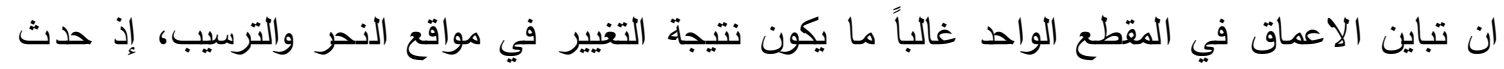
ترسيب على الجهات المحبة من الضفاف ونحر على الجهات المقعرة. وتختلف عمليتا النحر والترسيب بأختلاف لئل سرعة المياه والانحدار والمكونات الصخرية للضفاف على طول البحيرة التنظيمية. الكلمات الدالة: الهيدرولوجي، البحيرة التظيمية، المورفولوجي، مسبار قياس الاعماق، مسح نهري.

\section{Hydromorphological Study of Regulating Lake of Mosul DamNorth Mosul City, Iraq}

\author{
Asaad A.M. Al-Hussein \\ Dept. of Geography / College of \\ Education \\ Al-Hamdaniya University
}

\author{
Adil A.B. Al-Hamadani \\ Research Center For Dams And Water \\ Resources \\ Mosul University
}

\begin{abstract}
The work deals with the study of hydromorphology of the regulating lake which located at $(50 \mathrm{Km})$ north of Mosul city. The survey started in November, 2012 using the EchoSounder, and carried out by dividing the lake into (30) sections starting from the lower surface of the main dam and ending at the regulating dam. Data are recorded and represented using GIS technology (Arc GIs v10.4) and Microsoft program (Excel).

The survey shows that the shallowest depth in the lake is found at left bank of section $(5)$ which reached $(7.65 \mathrm{~m})$ and the deepest point is Found in the left bank of
\end{abstract}


the section (9) which reached $(18.22 \mathrm{~m})$ directly on the Thalwage line. The hydraulic gradient of the lake is $(1 \mathrm{~m} / \mathrm{Km})$, and the overall shape of the sections is (U) shape.

The variation in depth in each section is mostly due to difference in the sedimentation and erosion. There was deposition on the convex sides and erosion in the concave sides of the banks, the erosion and sedimentation processes vary with the variation of water velocity, slope and rock components of the river along the regulating lake.

Keywords: Hydrology, Regulating Lake, Morphology, EchoSounder, River survey.

\section{المقدمة}

تقع البحيرة التنظيمية لسد الموصل على بعد (50km) شمال مدينة الموصل بين خطي طول

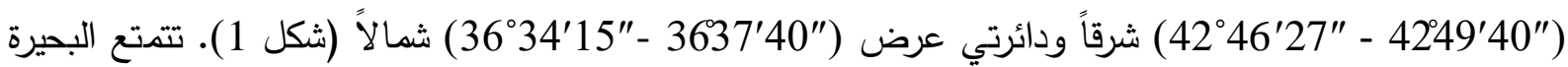
التظظيمية بأهمية خاصة وذلك لإنها تنظم التصاريف المطلقة إلى نهر دجلة وكذلك توفر المناسيب الملأئمة لتوليد الطاقة الكهربائية من السد التتظيمي. لإيه كانت البحيرة التظظيمية خلال فترة المسح (2012/11/22) تشابه مقطعا من نهر دجلة وذلك لان التصاريف المطلقة من السد الرئيس والسد التتظيمي كانت متساوية اعتماداً على البيانات المأخوذة من (إدارة مشروع سد الموصل). تم قياس طول البحيرة التنظيمية وبلغ (10.2km) ومساحة منطقة التغذية (95.68km²)

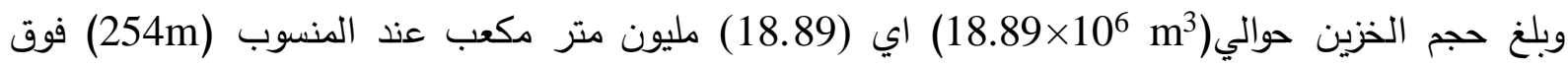
مستوى سطح البحر • يتميز هذا المقطع (البحيرة التظيمية) من نهر دجلة بتغاير منسوب الماء فيه بشكل مستمر بسبب تشغيل منظومة الخزن بالضخ (storage pump) والتي تسحب الماء من البحيرة التنظيمية وترفعه إلى مئ الخزان الاصطناعي في اعلى طية بطمة مما يؤدي إلى انخفاض منسوب البحيرة التتظيمية، ثم بعد ذلك وبسبب

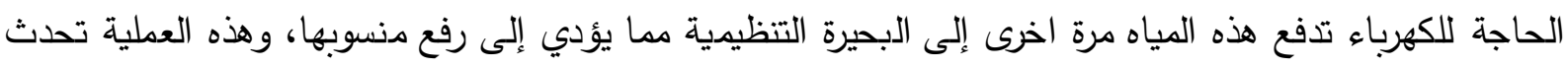
كل يوم تقريباً والتي لها تأثير مباشر على ضفاف البحيرة وأحد العوامل الرئيسة في انهيارها. إن منسوب البحيرة اسفل السد الرئيس يبلغ (254m)، اما قرب السد التنظيمي فهو (244m) فوق مستوى سطح البحر.

تركيبياً تتميز المنطقة بوجود طيتين محدبتين غاطستين غير متتاظرين هما طية بطمة ) (Anticline الغربي وعلى الغاطس الثرقي لها، والتركيب الثاني هو طية طيرة (Tairah Anticline) الواقعة في شرقيه، وهي عبارة عن طية محدبة غير متناظرة ثنائية الغطس يكون ميل طرفها الجنوبي بحدود (15) درجة في حين يبلغ ميل الطرف الثمالي (7) درجة (الحمداني، 1997). وتقع هاتان الطيتان على نفس الامتداد ويفصلهما

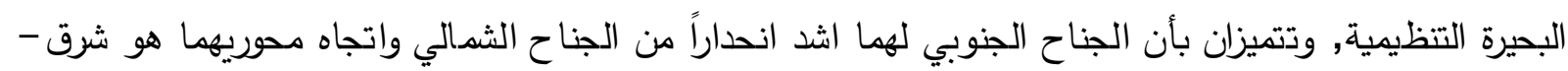
غرب (الجوادي, 1989). طباقياً تتكثف في منطقة الدراسة العديد من التكاوين الجيولوجية الممثلة للفترة الزمنية من(Lower Miocene) إلى (Recent), وكما موضح في الخارطة الجيولوجية (شكل 2), والتكاوين المتواجدة في منطقة الدراسة والمرتبة من الاقدم إلى الاحدث هي تكوين الفرات وتكوين الفتحة وتكوين انجانة وترسبات العصر الرباعي. 


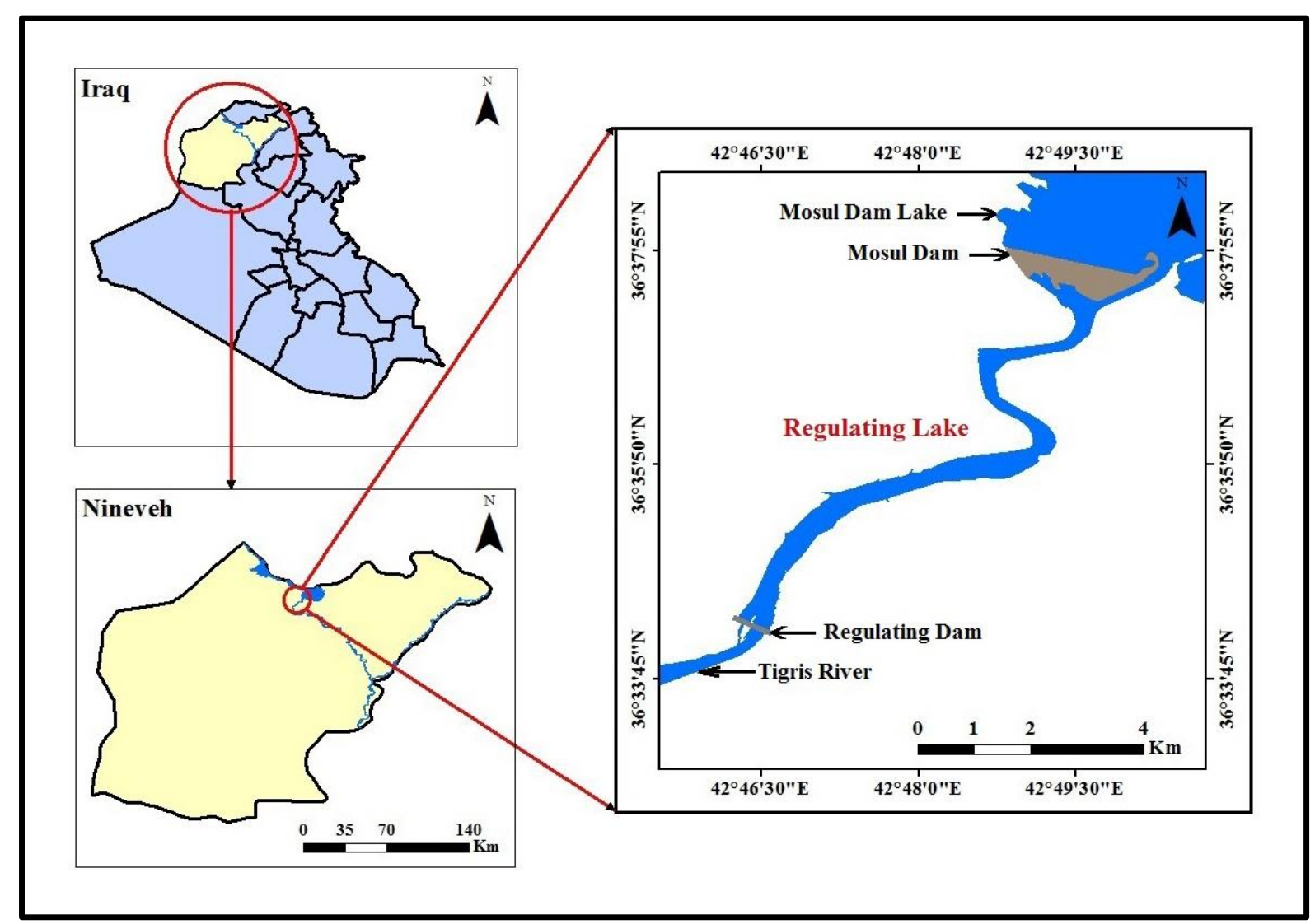

الثكل 1: خرطة موقعية لمنطقة الواسة.

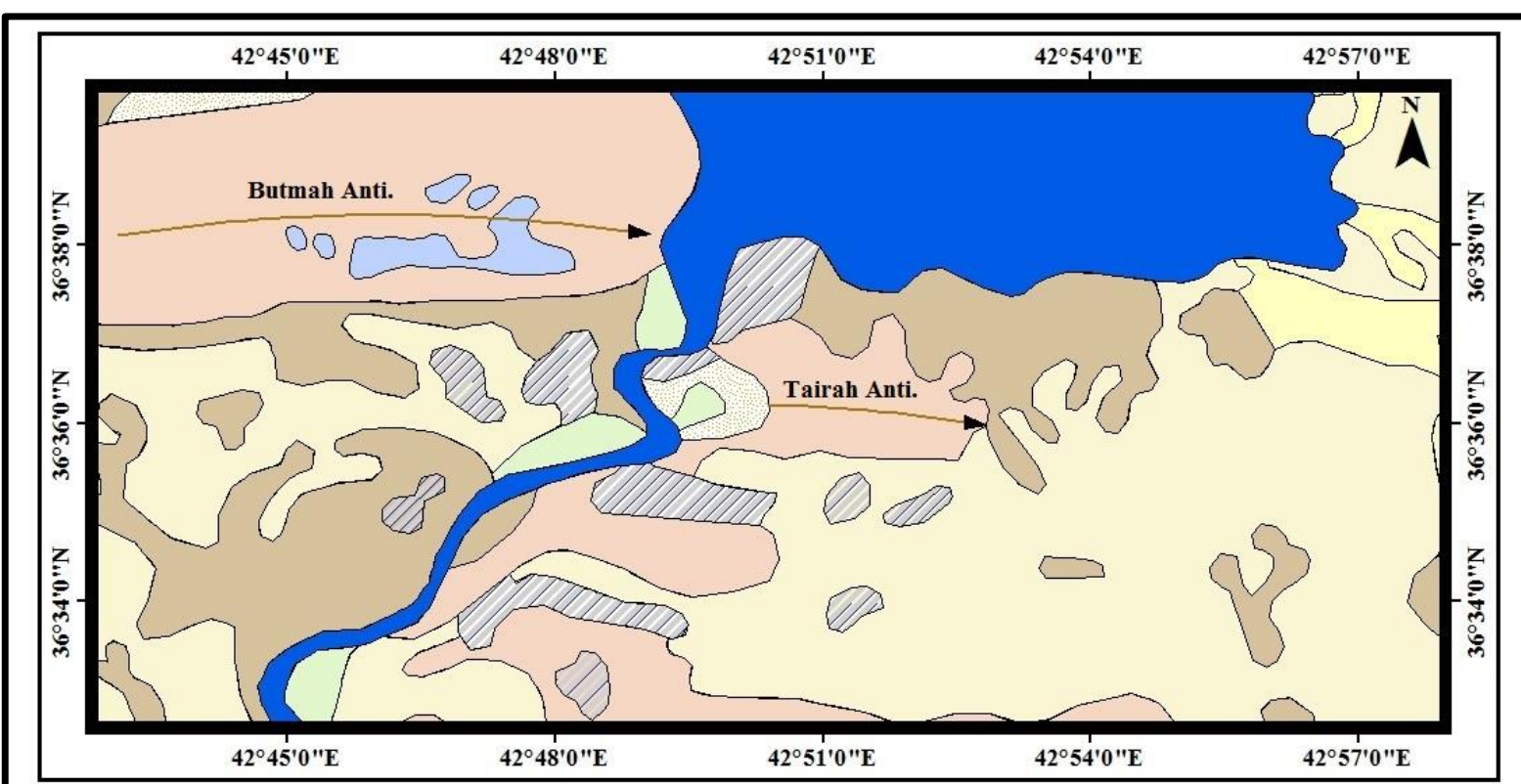

Legend

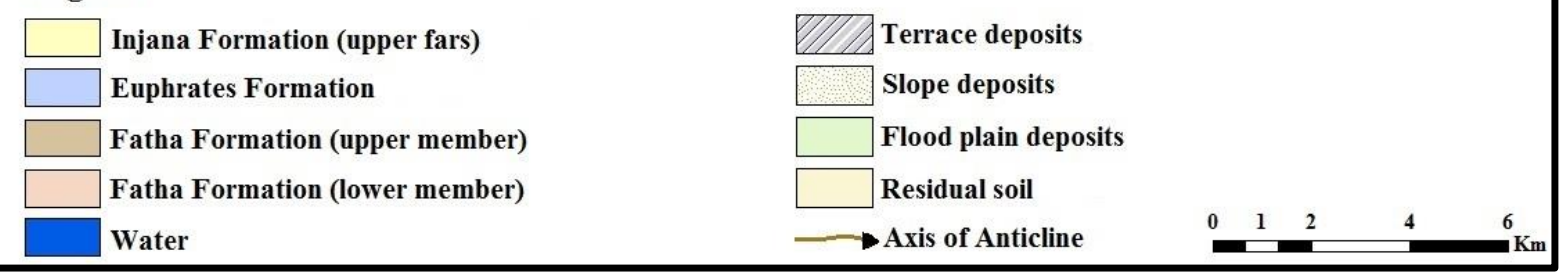

الثكل 2: خرطة جيولوجية لمنطقة الثراسة. 
تركيبياً تتميز المنطقة بوجود طيتين محبتين غاطستين غير متتاظرين هما طية بطمة الواقعة في غربي جسم السد, حيث أنشئ سد الموصل على جناح هذه الطية في الجانب الثمالي الغربي وعلى الغاطس الثرقي, والتركيب الثاني هو طية طيرة (Tairah Anticline) الواقعة في شرقيه, وهي عبارة عن طية محدبة غير متناظرة ثنائية الغطس يكون ميل طرفها الجنوبي بحدود (15) درجة في حين يبلغ ميل الطرف الثمالي (7) درجة (الحمداني، 1997). وتقع هاتان الطيتان على نفس الامتداد ويفصلهما

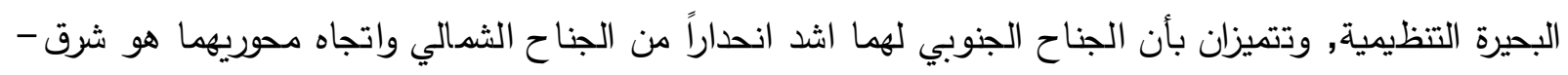
غرب (الجوادي, 1989). طباقياً تنكثف في منطقة الدراسة العديد من التكاوين الجيولوجية الممثلة للفترة الزمنية من (Lower Miocene) إلى (Recent), وكما موضح في الخارطة الجيولوجية (شكل 2), والتكاوين المتواجدة في منطقة الدراسة والمرتبة من الاقدم إلى الاحدث هي تكوين الفرات وتكوين الفتحة وتكوين انجانة وترسبات العصر الرباعي.

\section{طربقة اللعمل}

نفذت عملية المسح للبحيرة في شهر تشرين الثاني (20-2012/11/22), وتم الحصول على التصاردف المطلقة ومناسيب البحيرة خلال فترة المسح من إدارة مشروع سد الموصل وكانت التصاريف المطلقة من السد الرئيس (376m³/sec) ومن السد التنظيمي (373 m³/sec)، اي ان البحيرة تسلك سلوك النهر في هذه المدة. وكانت بداية عملية المسح على بعد حوالي (250m) عن التوربينات وحوالي (378m) (30m) عن المنفذ السفلي، وقسمت البحيرة إلى (30) مقطعاً عرضياً لتغطيتها بثكل كامل تقرببا إبتداءا من السد الرئيس وانتهاءاً بالسد

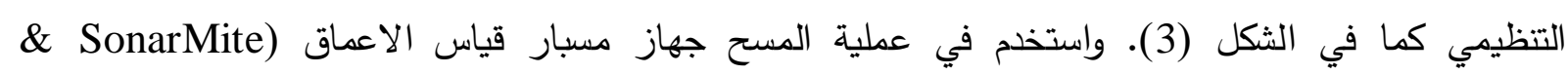
EchoSounder وجهاز (GPS) واوتاد وزورق للتحرك بشكل عمودي على المجرى اثثاء المسح. 


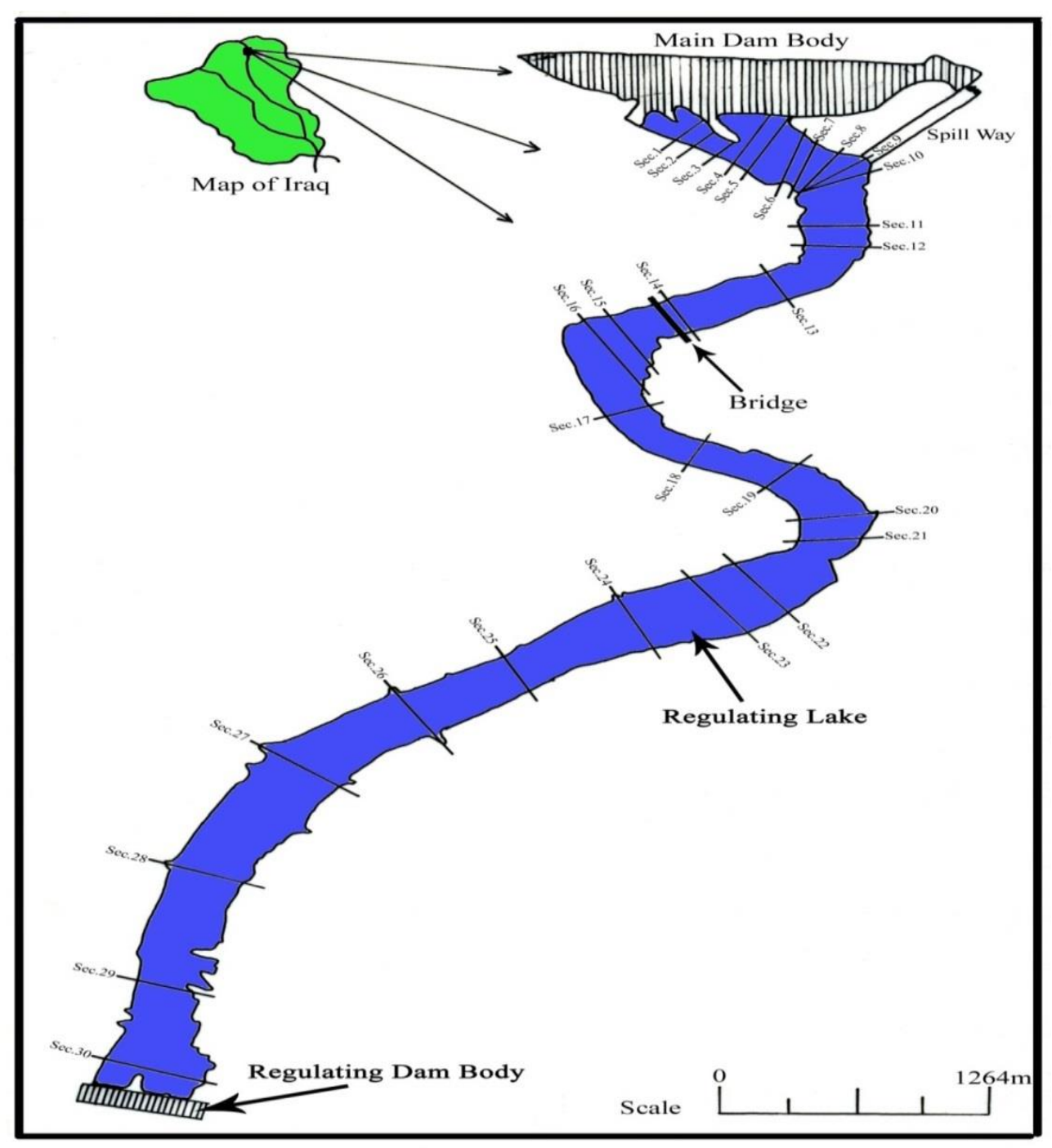

الثكل 3: المقاطع العرضية المدروسة.

\section{مورفولوجية ضفاف البحيرة}

بعد استطلاع شامل للبحيرة التنظيمية لوحظ ان الضفة اليمنى قليلة الانحدار في بداية البحيرة. وقد قامت الشركات المنفذة لسد الموصل بتسوية الأرض من اجل بناء إدارة مشروع سد الموصل وإدارة دائرة الكهرباء ومحطات توليد الكهرباء. وفي نهاية الثلث الاول من البحيرة تصبح الضفة اليمنى شديدة الانحدار بعد الجسر الارداء الحديدي وذلك لوجود مرتفعات تمثل جزء من الطية المقعرة ما بين طية بطمة وطيرة المحدبة وبعد ذلك تزداد الضفة اليمنى بالارتفاع حتى السد التتظيمي وهذه مرتفعات تمثل طية اخرى. اما الضفة اليسرى فتكون شديدة

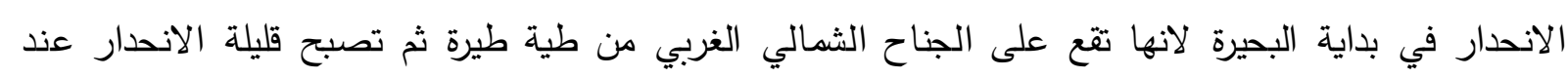

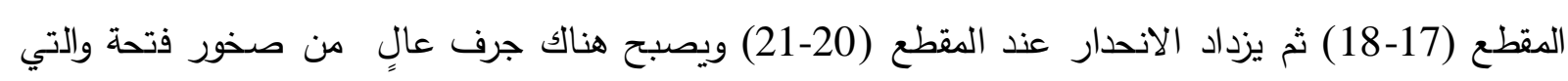
تمثل نهايات طية طيرة وبعد ذلك يقل الانحدار بأتجاه السد التنظيمي وتصبح المناطق المرتفعة بعيدة عن مجرى النهر • وقامت الفرق الهندسية الخاصة بإدارة مشروع سد الموصل بوضع احجار ذات احجام مختلفة تتراوح بين (1-0.5m) على الضفاف ضمن المقاطع العرضية (4,3,2,1) وذلك لغرض المحافظة عليها من التآكل والسقوط لئل 
لقربها من التوربينات والمنفذ السفلي لمنع تأثير المياه الخارجة من السد الرئيس والتي تكون ذات سرعة عالية وخالية من الرواسب مما يؤدي إلى تآكل الضفاف وجرف قاع البحيرة، وخلال مدة من الزمن زحفت الجزء السفلي من هذه الاحجار إلى مجرى النهر (الثكل 4), ثم عوض النقص لاحقا للضفاف بوضع احجار اخرى.

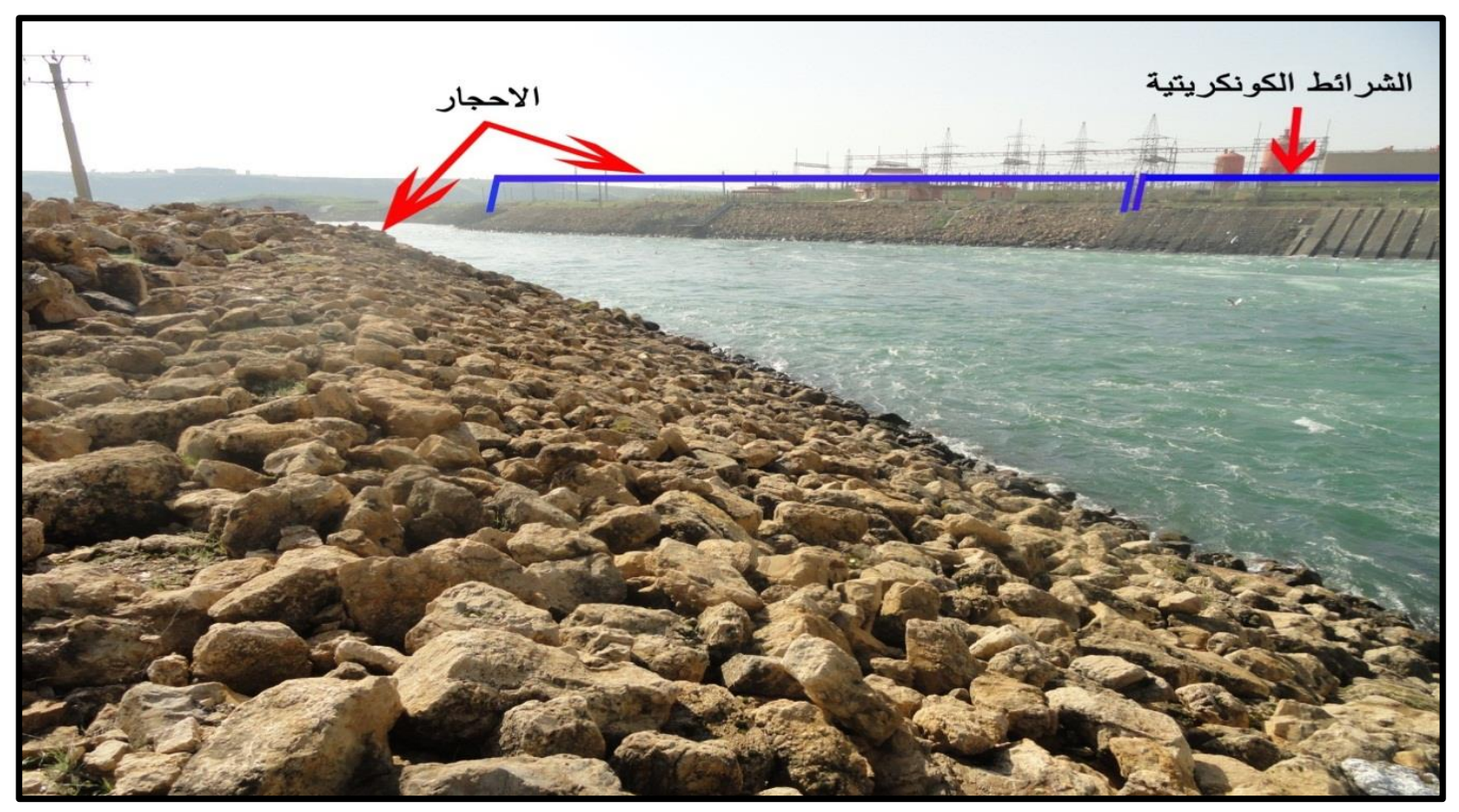

الشكل 4: الأحجار الموضوعة على ضفاف البحيرة.

\section{مورفولوجية البحيرة}

ادخلت بيانـات المسـح الحقلي التـي تم الحصـول عليهـا من اعمـاق المقـاطع العرضدية المدروسـة بيرنـامج وكذلك استخدم هذه البيانات في برامج خاصـة اخرى (SonarXP) على اشكال توضح خصائص الباثومتري لقاع البحيرة التنظيمية وكما في الثكل (5) والذي يوضـح توزيع الاعمـاق بشكل مختلف في البحيرة ، حيث تكون اعمق نقطة هي (18.22m) عند الضدفة اليسرى للمقطع العرضدي (9)

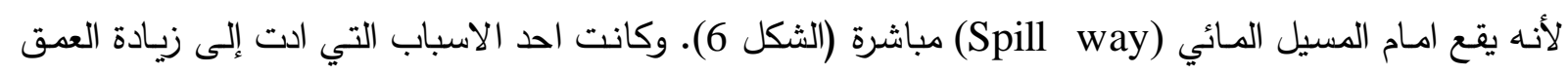
ونحر قاع البحيرة عند هذا المقطع هو تأثير اندفاع المساء بشكل كبير اثناء تشغيل المسيل المائي (الحمداني، 1997)، وأوطأ نقطة في البحيرة كانت عند الضفة اليسرى للمقطع (5) وبلغ (7.65m) وذلك بسبب قرب المقطع من تراكم مخفات نواتج التحشية للسد الرئيس (شكل 7)، وكذلك زحف ودحرجة الاحجار من قاع البحيرة للمقاطع

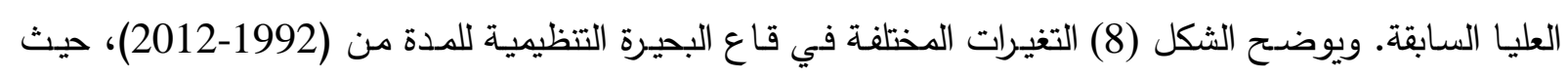
تكون الاعمـاق متباينة من مقطع إلى اخر ضدمن البحيرة، فالأعماق القليلة يعود سببها إلى اتساع نسبي لعرض فئره

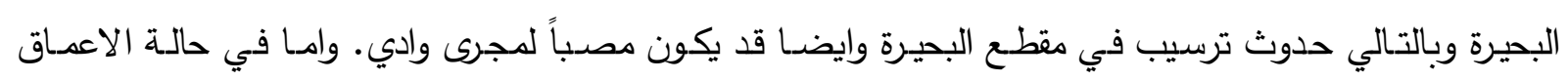

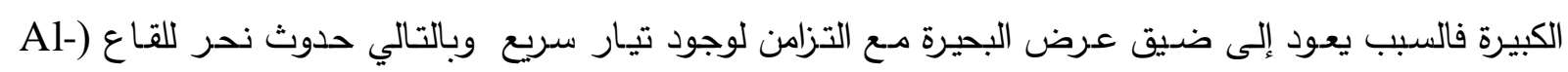

.(Hamdani et al., 1992 


$$
\text { اسعد احمد مقداد آل حسين و عادل علي بلال الحمداني }
$$

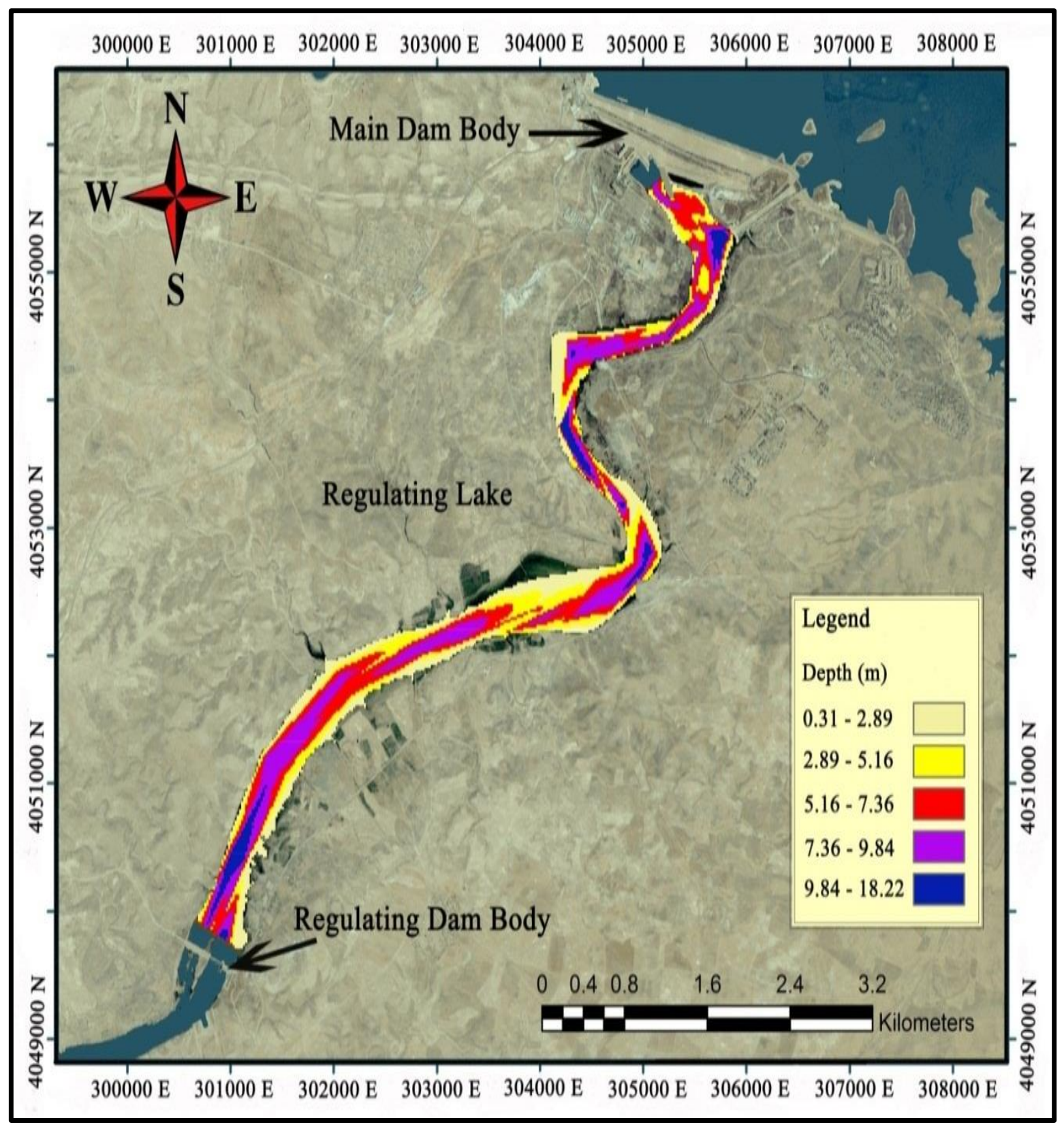

الثكل 5: اعماق المقاطع العرضية الموزعة على طول البحيرة التنظيمية.

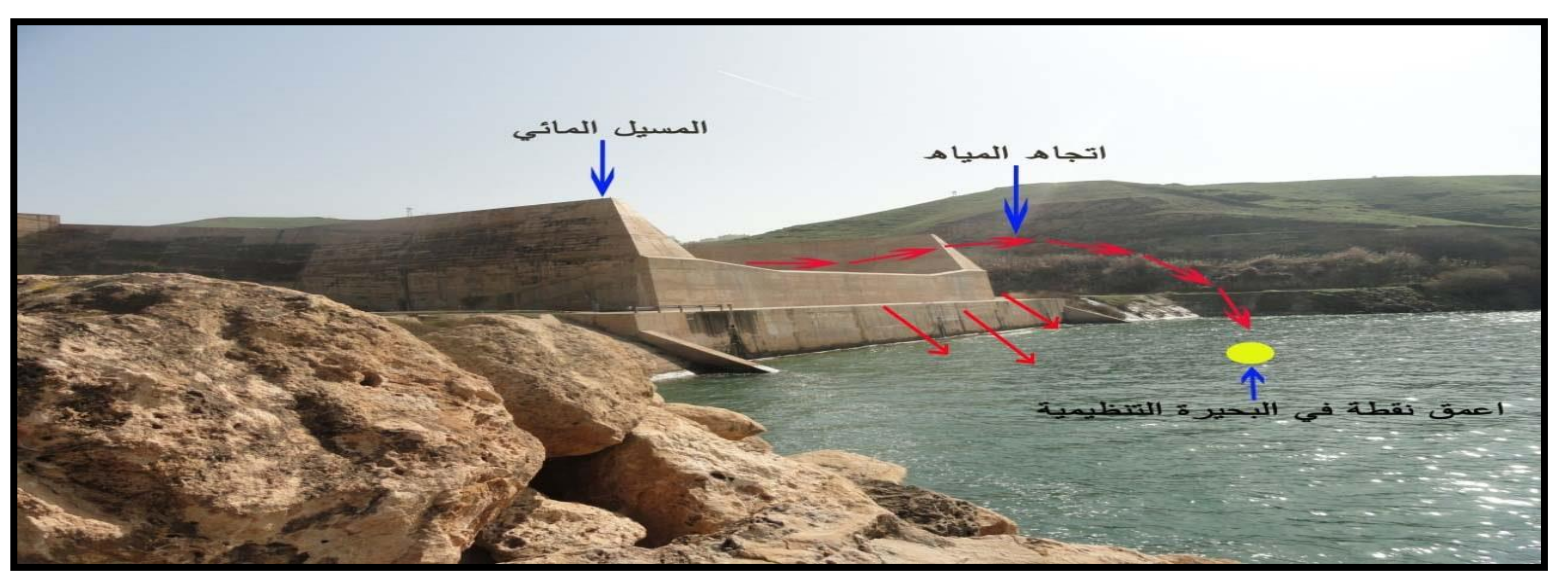

الثكل 6: المسيل المائي (Spill Way). 


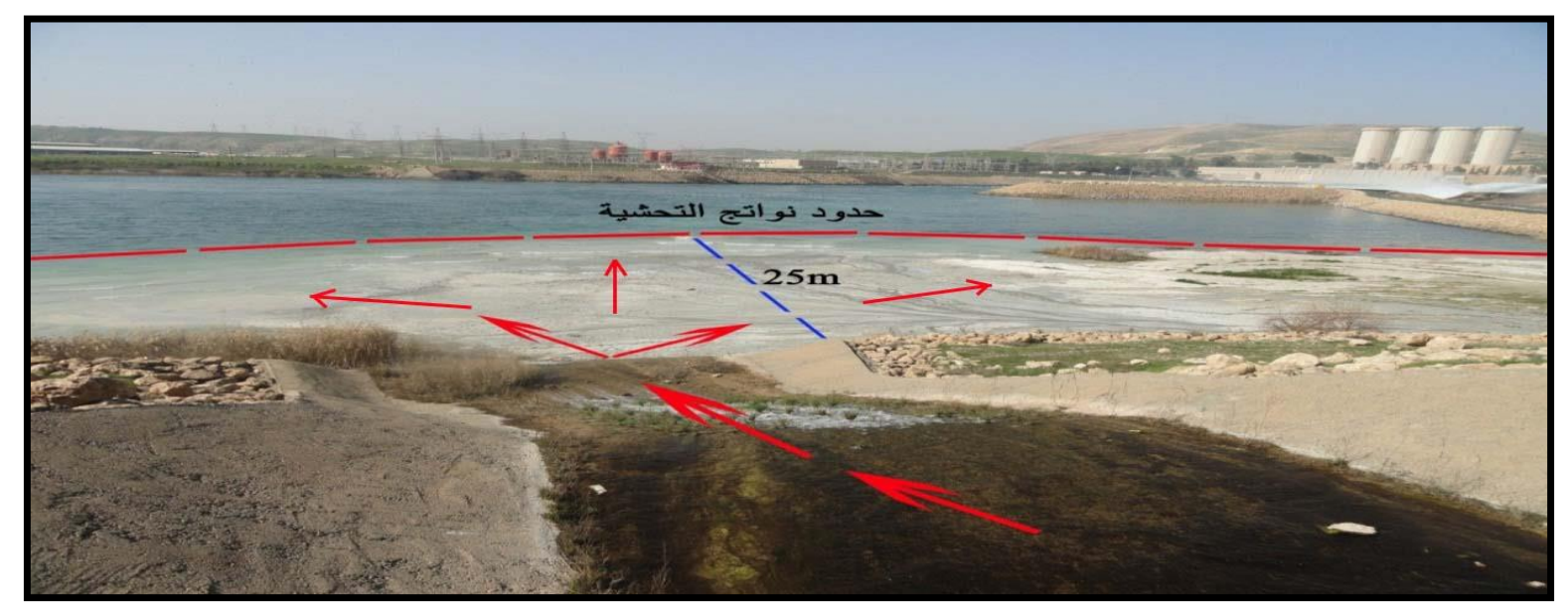

الثكل 7: فضلات تحشية قاع السد الرئيس.

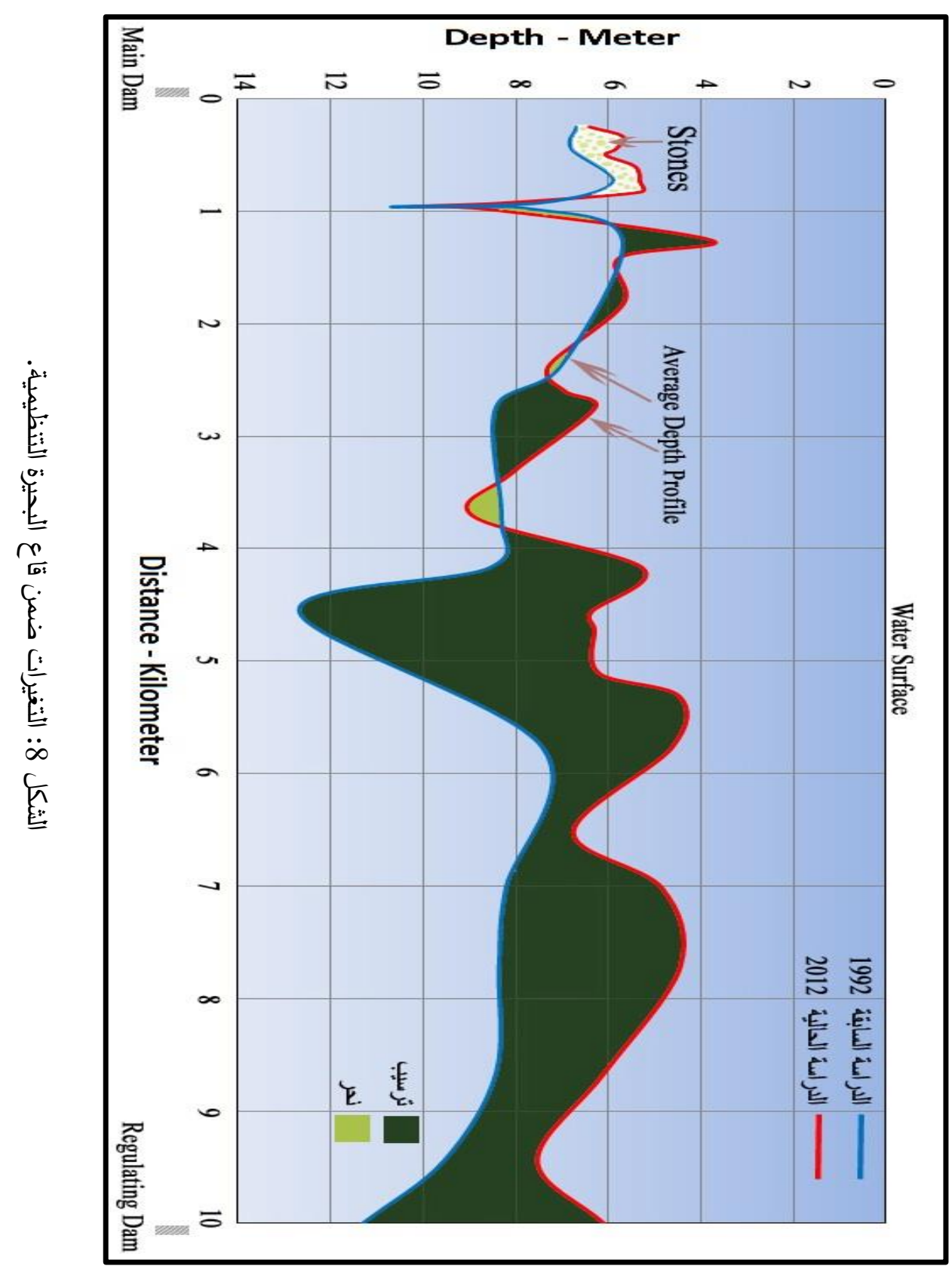




\section{المناقثثة}

تمت مقارنة هذه المقاطع مع دراسة (Al-Hamdani et al., 1992) على نفس المنطقة لمعرفة التغيرات التي حدثت خلال مدة (20) سنة، ولوحظ ان معدل الاعماق للمقاطع العرضية (7,6,5,4,3,2,1) انخفض مما فال يدل على حدوث ترسيب في هذه المواقع وهذا عكس المتوقع، لكن السبب الحقيقي في هذا الترسيب هو ان جزءاً كبيراً من الاحجار التي وضعت لمعالجة نحر الضفاف قد نقل إلى هذه المواقع . وتبين وجود نحر وخصوصاً أمام الضفة اليسرى للمقاطع (10,9,8) وذلك بسبب وصول دفق المياه المصرفة من المسيل المائي (Spill Way). يبدأ عرض البحيرة بالزيادة عند المقطعين (12,11) نتيجة الانهيارات التي حدثت في الضفة اليمنى أثناء تشغيل المسيل المائي في فترات سابقة، بينما لوحظ حدوث نحر عند المقطعين $(14,13)$ لان عرض البحيرة ضمن هذين المقطعين قليل بسبب انعدام تأثير المسيل المائي فضلاً عن صلابة الضفة اليسرى المتكونة من المتكتلات والتي تمثل جزءاً من طية طيرة.

إن بداية ظهور التواء مجرى البحيرة إلى جهة اليسار يكون ضمن المقطعين (16,15) نتيجة اصطدام مياه البحيرة بمرتفعات تمثل جزءاً من الطية المقعرة ما بين طية طيرة وبطمة المحبة فضلاً عن اتساع عرض البحيرة وحدوث ترسيب في الجهة اليسرى من المقطعين نتيجة قلة السرعة. ان مجرى البحيرة الرئيس يكون بالقرب من الضفة اليمنى للمقاطع (19,18,17) ومتوافق مع خط الثالوك، ولوحظ حدوث ترسيب في المقطعين (19,17) قرب الضفة اليسرى نتيجة الرسوبيات القادمة من الضفاف. إن المقطعين العرضيين (21,20) يمثلان موقعاً لانحناء مجرى النهر ولوحظ انه خلال مدة (20) سنة حدث ترسيب كبير ضمن المقطع (20) وان معظم الرسوبيات جاءت من مصب الوداي القربب من المقطع والذي يغذي البحيرة بالمياه أثناء موسم الأمطار، ومن

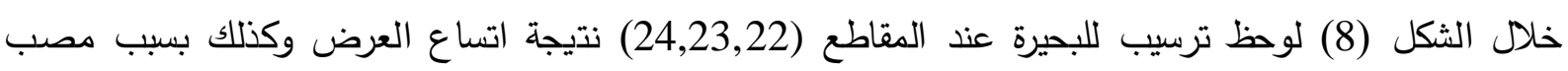
الوادي القريب، وكما لوحظ إن معدل الأعماق للمقاطع (28,27,26,25) انخفض والسبب هو الرسوبيات القادمة من الوديان التي تغذي البحيرة بالمياه ضمن هذه المقاطع. ولوحظ من خلال الثكل (8) ايضاً حدوث ترسيب للبحيرة ضمن المقطعين $(30,29)$ بسبب تأثير السد التنظيمي.

تكون اغلب المقاطع العرضية للبحيرة مشابهة إلى الحرف (U) (شكل 9)، وهذا يعود إلى توسع عرض

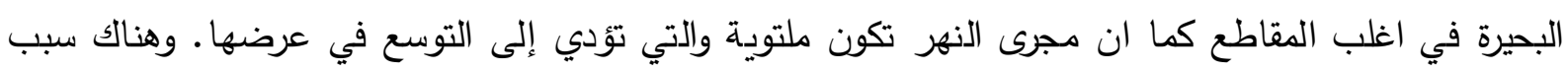
اخر قد يعود إلى الاختلاف في طبيعة وصلابة صخور الضفاف وصخور قاع البحيرة. 


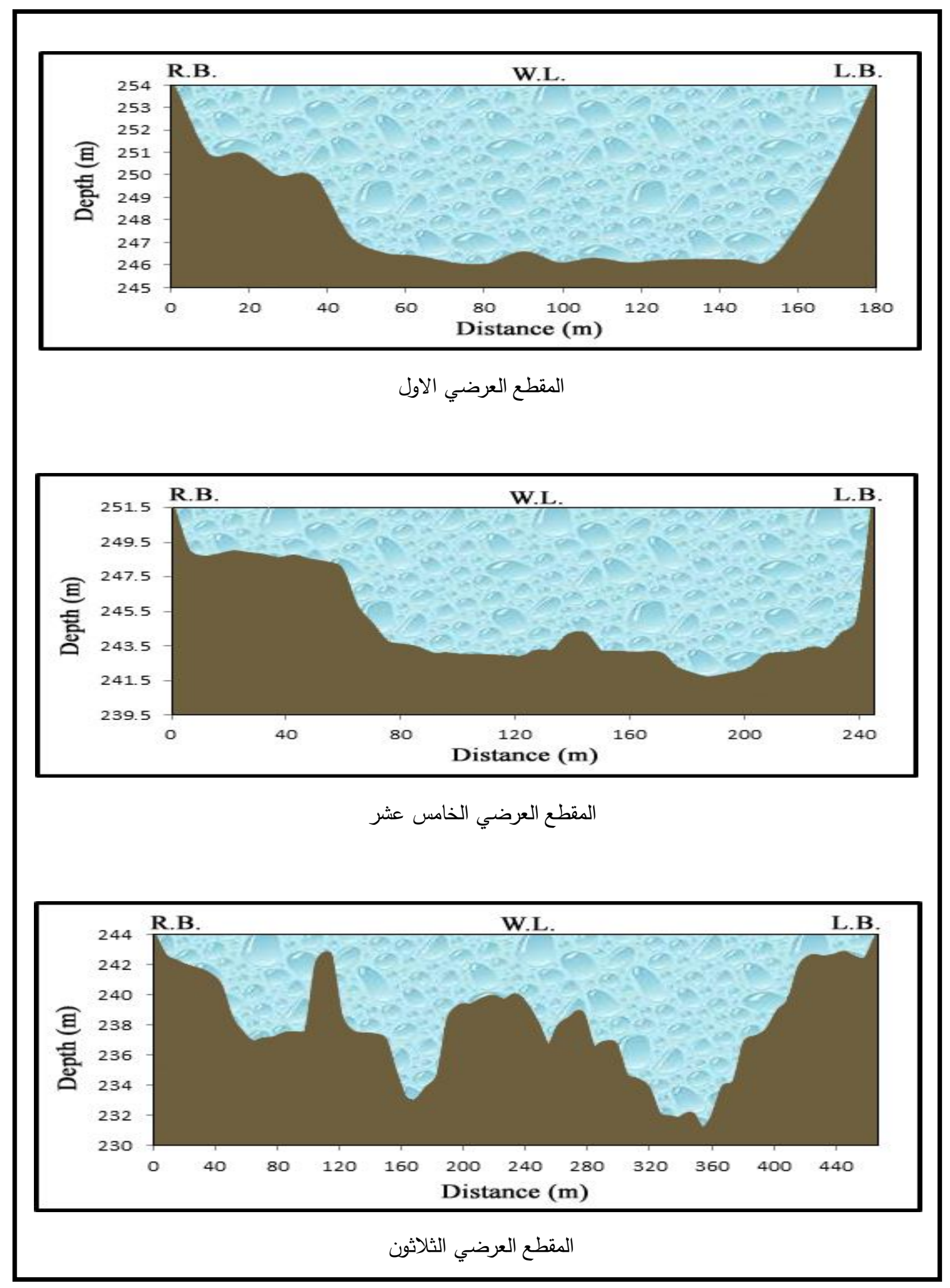

الثكل 9: بعض المقاطع العرضية المختارة من البحيرة التظظيمية. 


\section{الاستنتاجات}

1. يتراوح معدل الأعماق للمقاطع مابين (10.3-3.7m) واعمق نقطة وجد في الضفة اليسرى للمقطع العرضي الإن

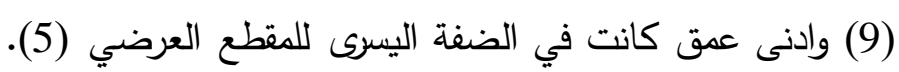

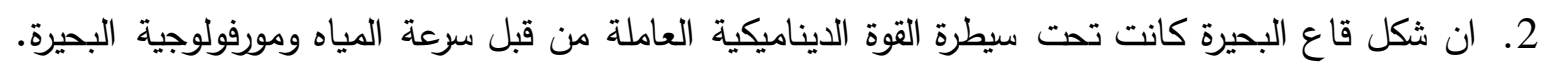

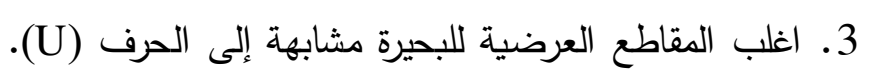

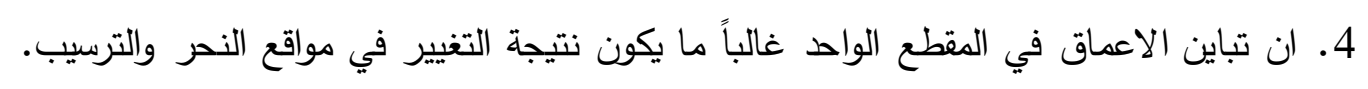

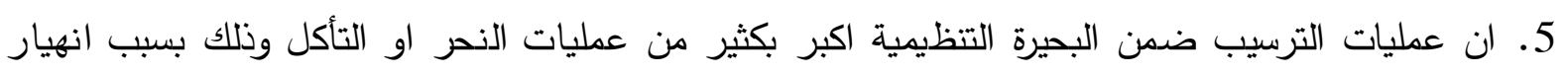

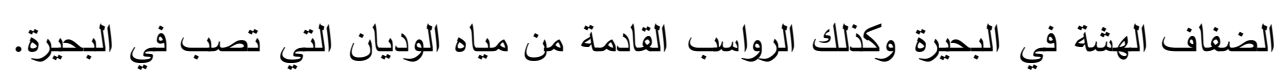

\section{التوصيات}

1. حماية ضفاف البحيرة التنظيمية المتكونة من مواد هثة من الانهيار بأساليب وطرائق مختلفة من خلال دراسة مفصلة لكل موقع.

2. العمل على زيادة الغطاء النباتي للتقليل من عميلة تعرية التربة من حوض التغاء لتغية. 3. 4. إزالة الترسبات المتراكمة في البحيرة بعملية الكري باستخدام تقنيات مختلفة.

\section{المصادر العربية}

الجوادي، عز الدين صالح، 1989. الصفات الجيوتكتونية والتصنيف الهندسي لصخور تكوين الفارس الاسفل

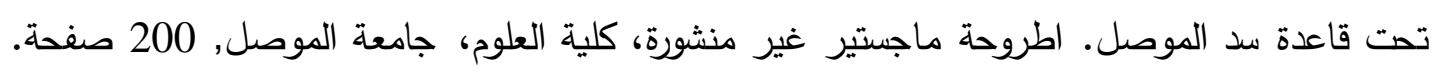

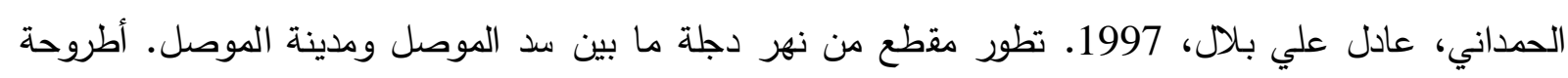

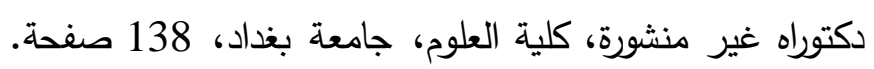
إدارة مشروع سد الموصل, 2012. التقرير اليومي لسلامة سد الموصل, وزارة الموارد المائية, الهيئة العامة للسدود والخزانات, العراق, بيانات غير منشورة.

\section{المصادر الاجنبية}

Al-Hamdani, A.A. Bilal, Al-Taiee, T.M. and Al- Ansari, N.A., 1992. Bed Characteristics and Morphology of Artificial Reservoir on Tigris River- North Mosul City, Jour. Water Resources, Vol. 11, No. 2., Pp. 45-55. 